\title{
Vehicular Traffic Monitoring Using VANET
}

\author{
Ms Shreya Sharma, Ms Rupali Rohankar \\ Computer Science, Amity University, Noida,UP \\ Shreyashrma444@gmail.com \\ Computer Science, Amity University, Noida,UP \\ rrohankar@amity.edu
}

\begin{abstract}
During last few years, continuous progresses in wireless communication have open new research fields in computer network. VANET proposes communication between vehicles by wireless means has a large potential to improve safety and travel comfort of passenger. Moreover automobile traffic safety is a major issue in developed area in which there may be misbehaving of traffic safety due to lack of controls or misguiding from the attacker end. Due to this, there is high amount of wastage of time and resources travelling through traffic congestion and misrouting of the vehicles by external source. For the sake of this, we propose an algorithm for VANET in which we show the effect of and further remove it by actual path routing and communication using distance algorithm to further improve the traffic pattern.
\end{abstract}

\section{Indexing terms/Keywords}

AODV Routing protocol, Distance algorithm, Traffic monitoring, traffic congestion, V2V and V2I communication, VANET .

\section{Academic Discipline And Sub-Disciplines}

Wireless Technology, Networking, Adhoc mobile networks,sensor networks

\section{SUBJECT CLASSIFICATION}

Computer Science Engineering and technology

\section{TYPE (METHOD/APPROACH)}

Literary review, historical analysis ,Experimental approach, Computer Simulation.

\section{Council for Innovative Research}

\author{
Peer Review Research Publishing System
}

Journal: INTERNATIONAL JOURNAL OF COMPUTERS \& TECHNOLOGY

\author{
Vol 13, No. 1 \\ editor@cirworld.com \\ www.cirworld.com, www.ijctonline.com
}




\section{INTRODUCTION}

Traffic congestions are formed by many factors some are predictable like road construction, rush hour or bottleneck.Some are unpredictable like accidents, weather and human behavior. Drivers unaware of congestion eventually join it and increase the severity of it. The more severe the congestion is the more time it will take to clear once the cause of it is eliminated [8]. The ability of a driver to know the traffic condition on the road ahead enables him or her to seek alternate routes saving time and fuel. When many drivers face, traffic congestions that are specifically those related to localized incidents such as accidents and temporary disruptions will be less severe and only the vehicle in the vicinity of the incident, at the time of incident, will be effected. Transition from the free flow to the congested traffic often occur at the highway spontaneously despite the fact that road could satisfy higher traffic demand. A congestion detection and dissemination system is being proposed using VANET that makes use of efficient communication channel ,maintains location privacy, and provide drivers about the real time information of traffic congestion over long distances. The system uses vehicles itself equipped with simple inexpensive devices as the gatherer and distributer of the information excluding the need of costly road infrastructures such as camera, sensors or any other external equipment .It focuses over the design and analysis of the key properties of the VANET and traffic control and monitoring enabled by the aforementioned technology. Here a strategy is presented to reduce traffic congestion using vehicle to vehicle communication. Periodically emitted signals are used to analyze traffic and warn other drivers about possible occurrences of traffic break down. The proposed strategy is analyzed via computer simulations.

\section{GENERAL ARCHITECTURE}

The vehicular networking is basically a wireless adhoc network which provide communication among vehicles by following manner:
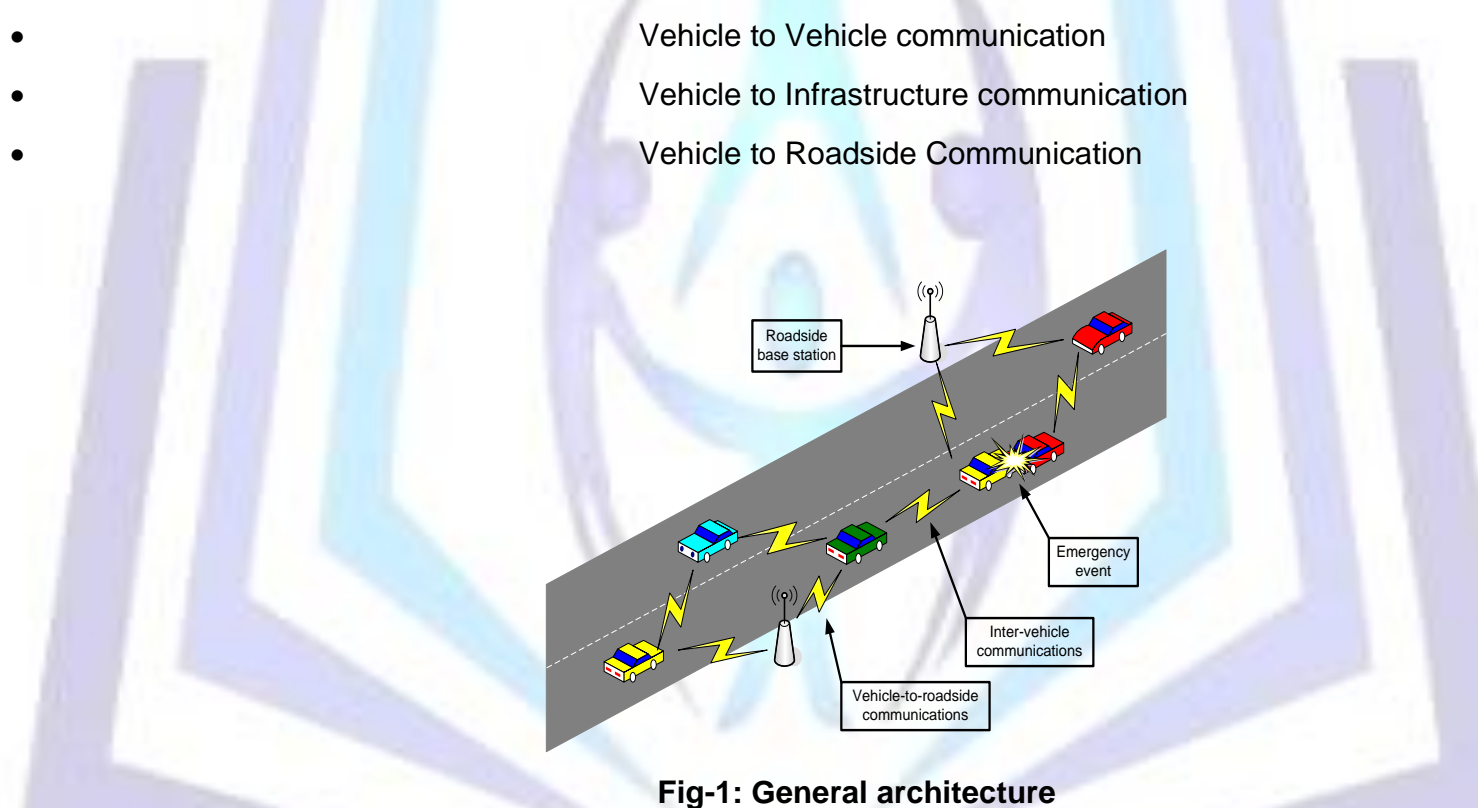

VANET consists of vehicles with onboard sensors and roadside units which provide communication from vehicle to vehicle or vehicle to infrastructure Each vehicle communicate with one another in a dynamic adhoc scenario The traffic related information can be moved all around the network from the periodic beacons which may include safety driven alert applications, infotainments including games and others.

\section{Factors influencing adoption of VANET}

Here we have discussed various factors which influence the users for the adoption of VANETS in the present scenario

1.Low Latency requirements for safety applications

Safety applications like collision alert, merge assistance, road condition warning etc requires messages to be propagated from the point of occurrence to the target vehicle with in few seconds with least latency .In contrast to the $3 G$ networks provide connectivity but have latency issues.

2.Extensive growth of the multimedia and interactive applications

Many applications like gaming and others require continuous internet connectivity for such we need to have a vehicle to infrastructure connectivity domains and VANET supports such features

3.Increasing concerns about privacy and security 
Although V2V communication may lead varietal security problem thus presence of V2I communication provide an efficient mechanism through centralized agencies can selectively broadcast the temporary identities with the public keys in the selective geographical area for the vehicles or the vehicles can pick up there required public keys in contrast to V2V where there occurs the problem with public key authentication.

\section{Current Solution to the Traffic Congestion}

Mostly current navigation systems are static and did not provide traffic information . Route selection is basically based on the static map data which leads to the system that fails to give driver most efficient route to his or her destination. In last many years these devices have incorporated real time traffic information systems to aid in route selection.

These real time systems like NAVTEQ [5][6] rely on human or any road infrastructure like traffic camera and radar to maintain a central database for traffic conditions. It there by limits its reach to major areas and thus reduces its utility. Initially in New York, 81 cameras were placed which provide only visual information that needs to be translated into congestion information either by humans or any specialized software which were only one way oriented where information is received either by fm radio or satellite. In order that vehicles could transmit data in real time to central infrastructure it may require an enormous communication infrastructure.

Two way communications such as Onstar [2] rely on cell phone communication which may not be always on and are not designed for a huge volume of communication as required by the traffic traveling across. Here we have proposed a technique where distributed approach is being utilized which uses vehicle themselves as a node in vehicular adhoc network as well as a data gathering devices that collect the necessary information to determine current traffic conditions and disseminate them over long distances using V2V communication, including such information into the vehicles navigation may provide driver with aware traffic routing using an efficient routing algorithm that takes into account traffic information. It is the most efficient approach as it provides a greater degree of flexibility and reduces the investments as cost is distributed among many drivers.

\section{RELATED WORK}

The Most of the research work in VANET focuses on simulating the vehicular traffic and multi hop routing .Some researchers are working in VANET to discover and disseminate traffic congestion problem [8] .Vehicle based GPS systems can be used in order to search and disseminate the traffic congestion information.

Collision avoidance systems[7][9] are designed which detect traffic incident in real time and convey such information to the nearby vehicles in order to prevent collision. But these systems were different from the traffic congestion systems as the information was to be conveyed over short distances and needs to be extremely reliable while in later the information is to be relayed remains current and thus need to be disseminated and used for congestion amelioration.

In recent year several researchers are into the issue related to distributed detection and dissemination of the information. Fukomoto et al[4] proposed a system which uses GPS systems for disseminating the traffic information, they called these systems as COC for VANET and this basically contains three info: Raw information(level1), Density information(level 2), and congestion area information (level 3). And higher levels may contain aggregated information.

Donrbush et al [3] from university of Maryland proposed a Street Smart system for congestion detection that uses clustering as a data aggregation technique to combine related recording of a unusually slow speed. StreetSmart uses clustering algorithm that works over a distributed network where each node analysis the collected statistics thus eliminating the need of central entity. Clustering is a process that groups together the data points which are similar to one another in some aspects or the other.

Yoon, Nobel and Liu of university of Michigan [10]proposed a traffic estimation system based on road segmentation and a inner traffic complexity scenarios, Some of these systems rely explicitly or implicitly on receiving the location from the vehicles in order to detect either the congestion is there or not. when the size of congestion exceeds a particular transmission range which generally occurs in freeway scenarios ,the multi hop communication come into existence which concludes a problem whenever we want to keep location information anonymous a vehicle may receive fresh information and rebroadcasted information from the same vehicle, as vehicles move constantly these two pieces of information will be undistinguishable. Thus for this reason systems rely on unique IDs as a mechanism to identify source of each piece of information and maintain a unique location for each vehicle.

The broadcasting of unique vehicle IDs at the application level facilitates location tracking and thus leading privacy concerns. Recently many organizations in US have realized the importance of vehicular communication, Dash Navigation, Inc [1 ][2] a start up in sunny valley in 2009 provided services to allow drivers to broadcast their speed and location in exchange of receiving updated traffic information compiled from other drivers in the network. This is a centralized system and relies on wireless internet connectivity which is not globally available everywhere as the central authority concerning is trusted location and privacy concerns are thus mitigated. Traffic view focuses on the estimating the road congestions

\section{VANET}

Vehicular Adhoc network is a technology that uses moving nodes in the network to create a mobile network. VANET turns every participating node into a wireless router or node allowing vehicles to connect one another. As the vehicles drop out of a particular range other vehicles can join in connecting vehicles to one another so that a mobile internet is created. 
VANET is basically a subgroup of MANET where nodes refer to a vehicle. Network. VANET turns every participating node into a wireless router or node allowing vehicles to connect one another. As the vehicles drop out of a particular range other vehicles can join in connecting vehicles to one another so that a mobile internet is created. VANET is basically a subgroup of MANET where nodes refer to a vehicle.

\section{AODV routing protocol}

AODV is an on demand algorithm as it builds routes among nodes only when desired by the source node. And maintains such routes only uptill needed by the source node else it disconnects. It basically uses sequence number to ensure freshness of the route .It is a loop free, self starting and it easily scales to large number of mobile nodes. It bilds routes using route request/reply query cycle.When a source node desires a route to a destination for which it doesnot have a route prerequisitly, It broadcasts a route request packet across the network,Nodes which receives such packet update the information of the source and setup backwards pointer to the source in the roting table.In addition it may also contain source nodes IP address, current sequence number, and broadcast ID ,The RREQ also contain most recent sequence number for the destination of which source node is aware.A node receiving RREQ send a route reply(RREP) if it is either a destination or correspondant to it.Nodes keep track of RREQ source IP address and broadcast ID If they receive RREQ which is already present then the nodes are being discarded.As the RREP propogates back to the source node setup a forward pointer to the destination.

\section{PROPOSED WORK}

VANET has been studied in many fields since it has the ability to provide a variety of services such as detecting oncoming collision and providing warning signal alerts to the driver. The services provided by VANET are often based on collaboration among vehicles that are equipped with relatively simple motion sensors and gps units. It has been proposed as an automated solution to many problems related to transportation , highway safety and driving assistance . VANET facilitate by providing a communication among vehicles, Here our main focus is to build a communication scenario depicting vehicle to infrastructure and vehicle to vehicle communication. Sometimes there may arise situations when the vehicles receiving the signal may or may not receive the signal information as the may not have any specification assigned when to communicate and up to which distance the signal strength is most effective. Sometimes when vehicular density raises at an instance on a particular intersection there may occur packet loss because of network congestion. In order to overcome such problem we have proposed a distance algorithm. The algorithm eliminates the congestion by providing a fixed distance when and where information exchange can occur between the vehicle and the signal, thereby using vehicle to infrastructure communication. The distance being fixed between the vehicle and signal is Euclidian distance. Signal information is being send or received only after the vehicle reaches the Euclidean distance which is being measured for nodes in $\mathrm{x}$ and $\mathrm{y}$ planes. Where nodes serve as position of a vehicle. Euclidean distance between vehicles provide the idea regarding time. When a particular vehicle stops at a location with a speed we can easily depict the time. For instance we have fixed a timer at signal for 5 minutes, then the vehicles arriving at the distance of communication may receive the information and will wait till the signal changes from red to green, allowing the vehicles in the correspondent location to move.

The Euclidean distance algorithm is given for a vehicle

In order to find the Euclidean distance between two nodes $P$ and $Q$, first of all $P$ and $Q$ are described with coordinates $(p 1, p 2)$ and $(q 1, q 2)$ respectively. In first step length between the $P$ and $Q$ is given by $|p 1-q 1|$ and $|p 2-q 2|$. Secondly the Pythagorean Theorem is between the two length gives $\left((p 1-q 1)^{\wedge} 2+(p 2-q 2)^{\wedge} 2\right)^{\wedge}(1 / 2)$.

So the distance between two points $P=(p 1, p 2)$ and $Q=(q 1, q 2)$ in two dimensional space is there given as

$$
\left.\left.\left((P 1-q 1)^{\wedge} 2+(p 2-q 2)^{\wedge} 2\right)^{\wedge} 1 / 2\right)\right)
$$

Here we will fix a range of particular Euclidean distance from the signal to the vehicle and thus when the vehicle reach a particular Euclidean distance which is stored in the database the vehicles will start communicating and thus they get acknowledged about the further vehicular movements, the distance will depend on vehicular density and the time duration of vehicles travelling the particular area of intersection and thus we will propose a mechanism to find such a distance where vehicle to infrastructure communication may occur in order to prevent congestion

AODV routing algorithm is being used, AODV is the routing protocol designed for mobile adhoc networks. It is capable of both unicast and multicast routing .It provides a quick adaption to the dynamic link conditions, low processing and memory overhead. It discovers routes as and when necessary and doesn't maintain route from every node to every other node. Here nodes are maintained only when necessary, every node maintains its monotonically increasing sequence number which increases every time the node notices change in its network topology.It uses destination sequence number to ensure loop freedom at all time. ADOV maintains routes as long as the route is active. 


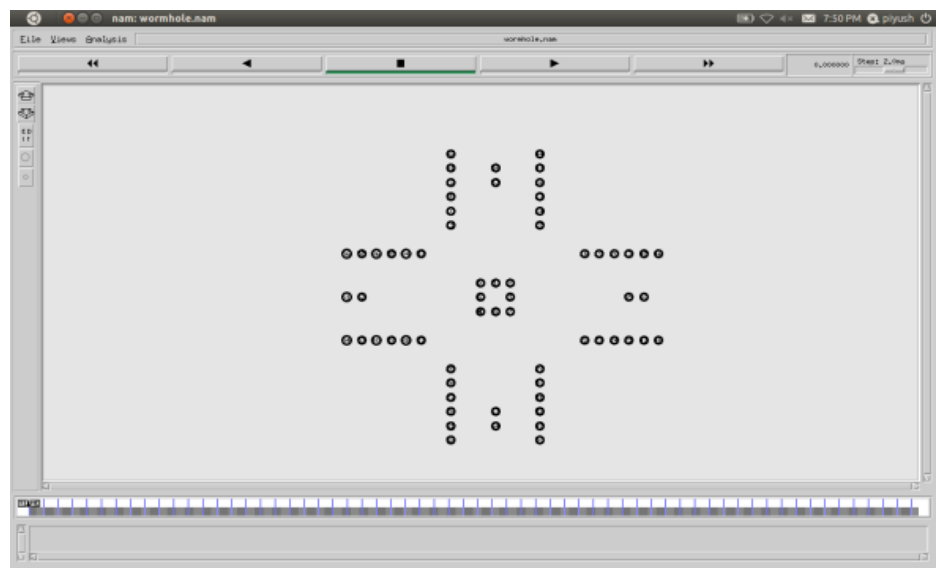

Fig:2 Simulation model

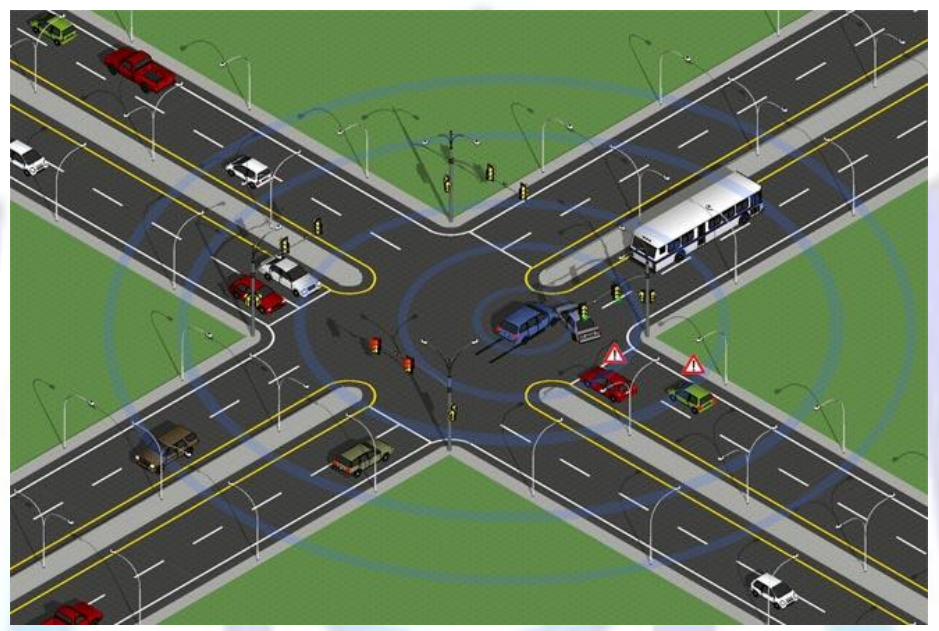

Fig :3 Vehicular Communication at Intersection

\section{ADVANTAGES}

The key advantages of the proposed system is that good network mainly concerns with efficient transfer of data ,minimizes cost, less packet loss, and reduces network overload.VANET systems offer some unique characteristics which are :

- Vehicles move at high speed

- Mobility pattern is predictable as the movement is constraint to the road infrastructures.Sometimes at highway mobility pattern becomes highly unpredictable.

- Large coverage area as vehicle move over a long distance and traffic information may be useful to the vehicle miles away.

- Here power consumption is not a major concern as vehicles itself can produce power

- Vehicles have a high cost and thus can be equipped with some additional sensors which may not significantly effect the total costs.

- VANET topology is highly dynamic as vehicles go in and out transmission range very rapidly

\section{CONCLUSIONS AND FUTURE WORK}

We have successfully reviewed various work done in vehicular adhoc networks and thus we have proposed a solution to avoid packet loss which may occur when highly crowded vehicular traffic gets accumulated at an intersection leading signal information loss thus causing miscommunication in order to prevent such we have introduced a significant distance algorithm where the communication may occur only when vehicle reaches a Euclidean distance from the signal and thus receive the information for the vehicular movement. The routing protocol for VANET being used is AODV.

The work presented uses a distributed approach that uses vehicles themselves as nodes in a vehicular adhoc network as well as data gathering devices to collect essential information in order to determine current traffic conditions and thus disseminate it over long distances using V2V communication. A distributed approach is the most efficient approach as it 
provides a greater degree of reliability and flexibility also it does not require a great investment over infrastructure as cost is being distributed among many vehicles acting as devices.

We will simulate the above scenario using AODV routing protocol using network simulator NS2. The results will be plotted in graphical form which will provide a comparison and also deliver the effectiveness of using distance algorithm in contrast when we may come across packet loss due to high density

\section{REFERENCES}

[1] Car2Carcommunication consortium.www.car2car.org [Online].

[2] DashExpress: The first two-way, Internet-ConnectedGPSnavigation system.[online].www.dash.net.

[3] Dornbush,S.and Joshi,A. Street-smart Traffic: Discovering and disseminating Automobile Congestion using VANET.

[4] Fukomoto,junya,et al.Analytic: Method for Real time traffic problems by using Content oriented communication in VANET. $7^{\text {th }}$ international conference on ITS ,2007,pp.1-6

[5] NAVTEQ Real time Traffic service [online]www.navteq.com.

[6] NAVTEQ Real time traffic service.

[7] Rawashdeh,Z.Yand Mahmud,S.M.Intersection Collision avoidance System Architecture System architecture $.5^{\text {th }}$ IEEE Consumer Communication and Networking Conference, 2008,pp.493-494Brown, L. D., Hua, H., and Gao, C. 2003.

[8] Scharank,David and Lomax, Tim :The 2007 Urban mobility report Technical Report s.I.: Texas Transportation Institute

[9] VANETwikipedia.org[online]en.wikipedia.org/wiki/

[10] Yoon,Jungkeun,Noble,Brian and Liu,Migyan. Electrical Engineering and computer science ,University

\section{Author' biography with Photo}

